46 patients (35\%) with 12 of $16(75 \%)$ having infectionrelated renal dysfunction, 2 (12.5\%) with hypovolemia, $2(12.5 \%)$ with HRS. Most of the mortality occurred in alcoholic cirrhosis (14 patients).

Conclusion: The most common cause of renal dysfunction was infection which led to a high mortality in patients with cirrhosis.

36

\section{PREVALENCE OF HEPATITIS E VIRUS INFECTION AMONG PREGNANT WOMEN IN RAJASTHAN}

\author{
V Bharadia, N Singh, S Nijhawan, RR Rai \\ Sawai ManSingh Medical College and Hospital, Jaipur, India
}

Background: Hepatitis E virus (HEV) is highly endemic in developing countries with high mortality rate among pregnant women. The prevalence of antibodies and RNA to $\mathrm{HEV}$ in Rajasthan is not known.

Aim: To evaluate the prevalence of hepatitis E infection among pregnant women at the SMS Medical College and Hospital Jaipur, Rajasthan, India.

Method: Pregnant females attending antenatal clinic attached to SMS Medical College between January 2010 and December 2010 who required blood sample for hemoglobin and blood group analysis were anonymously tested for the presence of anti-HEV IgG, anti-HEV IgM, and HEV RNA.

Result: Two-hundred and thirty-six pregnant women provided blood samples for unlinked anonymous testing for the presence of antibodies to HEV. The median age of participants was $28.89 \pm 5.76$ years. Among 236 women tested, HEV seroprevalence was $28.81 \%$. Among the seropositive women, $64.40 \%$ tested positive for anti-HEV IgM while $35.60 \%$ tested positive to HEV IgG antibodies. HEV seroprevalence was highest (46.15\%) among women $21-25$ years of age, followed by $42.82 \%$ in $<20$-year group, then $36.84 \%$ in $>26$-year group. Of 236 women, $75.79 \%$ and $22.92 \%$ were in their third and second trimesters of pregnancy, respectively. Anti-HEV antibodies detected in women in their third trimester of pregnancy $(30.25 \%)$ was significantly higher, $P<0.05$ than in women in their second trimester of pregnancy $(25.0 \%)$. Of $236,29.20 \%$ were positive for $\mathrm{HEV}$ RNA.

Conclusion: The results of our studies revealed a high prevalence of HEV infection in pregnant women.
37

\section{RETROSPECTIVE STUDY OF ISOLATED HEPATIC INJURY}

N Mehta, H Trivedi, S Parhad

Department of Surgery, MP Shah Medical College, Jamnagar, India

Background: During the last decade, there has been a change in the therapeutic protocols related to liver trauma, and surgery is no longer the only option available.

Aim: The aim of this study was to analyze the effectiveness and the morbidity-mortality of both conservative vs surgical treatment in a series of 50 patients with isolated hepatic injury during the last year.

Method: In this retrospective study of 50 patients, variables analyzed included demographic data, injury classification, surgical treatment, transfusions, morbidity-mortality, and hospital stay. The liver trauma was classified according to the Hepatic Injury Scale of the American Association for the Surgery of Trauma. Grade I: 8 cases; grade II: 14 cases; grade III: 20 cases; grade IV: 6 cases; and grade V: 2 cases. Of 42 patients from grades 1 to 3,37 patients were managed conservatively with the mean hospital stay of 9 days without any complications with the mean blood transfusion of 2 units. Rest of the 5 patients had to be operated due to failure of conservative trial. Five patients from grade 4 were treated conservatively with successful results. Three patients with grade 4 and 5 liver trauma were operated with $66 \%$ mortality rate. Surgical technique included limited resection-debridement, live vascular ligation, and perihepatic packing.

Result: If the right determinants with accurate diagnosis are clearly defined for isolated hepatic trauma, conservative management for grades I, II, and III is the best treatment option while grades IV and V need further evaluation.

\section{8}

\section{HEPATIC RESECTION-SCOPE FOR GENERAL SURGEON!}

D Vasavada, S Mehta, S Parhad

Department of Surgery, MP Shah Medical College, Jamnagar, India

Background: Hepatic resection has evolved over 50 years. In India, every general surgery department has a dream for successful liver resection, in spite of the scarcity of sophisticated instrument for liver resection such as Cavitron ultrasonic surgical aspirator (CUSA), argon beam coagulator, hydro-jet dissector, and harmonic scalpel. These devices are available only in few well-set gastrointestinal and hepatobiliary pancreatic surgical units.

Aim: Liver resection is possible by understanding of hepatic anatomy and precise technique. 